\title{
Heterogeneity in Spinal Bone Mineral Density Among Young Adults From Three Eastern Provincial Capital Cities in Mainland China
}

\author{
Xiao-Guang Cheng, ${ }^{1}$ Kai Li, ${ }^{1}$ Shan-Xing Ou, ${ }^{2}$ Guang-Yu Tang, ${ }^{3}$ \\ Qian-Qian Wang, ${ }^{4}$ Chao Wang, ${ }^{4}$ Ling Wang, ${ }^{1}$ and Wei Tian*,I
}

Q2 as ${ }^{1}$ Beijing Jishuitan Hospital, Beijing, China; ${ }^{2}$ Guangzhou General Hospital of Guangzhou Command, Guangzhou, China; ${ }^{3}$ Affiliated Tenth People's Hospital of Tongji University, Shanghai, China; and ${ }^{4}$ Beijing Institute of Traumatology and Orthopedics, Beijing, China

\begin{abstract}
This study compares spinal volumetric bone mineral density (vBMD) with spinal areal bone mineral density (aBMD) among young adults from 3 eastern provincial capital cities in Mainland China. A total of 416 young adults (age range: 20-40 yr) from 3 eastern provincial capital cities (Beijing, Shanghai, and Guangzhou) in Mainland China were recruited in this study. From each subject, the vBMD of the lumbar spine was measured by the Mindways quantitative computed tomography system. Moreover, the aBMD of the lumbar spine, measured by the dual-energy X-ray absorptiometry, was extracted from a previous multicenter large-scale study, and the 420 participants were matched by age, gender, height, weight, as well as geographic territory. The vBMD and the aBMD values were further compared and analyzed. Generally, the BMD results were significantly different among participants from the 3 cities $(p<0.05)$. Specifically, both vBMD and aBMD values of participants from Beijing were significantly different from those from Guangzhou $(p<0.05)$. Additionally, a statistically significant difference in aBMD values was also found between participants from Beijing and Shanghai $(p<0.05)$. However, no significant differences were found between participants from Shanghai and Guangzhou in terms of the aBMD and vBMD values $\left(p_{1}>0.05\right.$ and $\left.p_{2}>0.05\right)$. Interestingly, the overall mean vBMD value was $5.9 \%$ greater in women than those in men for all the 3 cities $(p<0.001)$. This study demonstrated an overall heterogeneity in spinal BMD among young adults from 3 eastern provincial capital cities in Mainland China. Specifically, the taller and heavier young adults from the northern part of China have smaller spinal vBMD but higher spinal aBMD values than those who were shorter and lighter from the southern part of China.
\end{abstract}

Key Words: Bone mineral density; dual-energy X-ray absorptiometry; osteoporosis; quantitative computed tomography.

\section{Introduction}

According to the 2012 Census Report of China, 14.3\% (194 million) of the Chinese population were older than

Received 10/24/15; Revised 03/20/16; Accepted 03/23/16.

*Address correspondence to: Wei Tian, MD, Department of Spine Surgery, Beijing Jishuitan Hospital, 31 Xinjiekou East Road, Xicheng District, Beijing, 100035, China. E-mail: xiao65@263.net
$60 \mathrm{yr}$, which would increase to $17.17 \%$ (248 million) by the year 2020. With the aging population, the prevalence of osteoporosis-related fractures, including spine, hip, and forearm fractures, might increase dramatically, leading to potentially increased morbidity and mortality (associated with hip fracture) as well.

Patients at high risk of osteoporosis should be identified and treated early to prevent fractures. Osteoporotic fracture results from loss of bone strength, which often 
happens during the precipitating fall or the protective actions (e.g., forearm extension) taken by a falling person. Bone strength relates to the function of bone quality, bone density, and bone geometry. However, because bone quality cannot be assessed in vivo, bone mineral density (BMD) is therefore considered to be a surrogate for bone strength, which is now regarded as the most important measurement for predicting osteoporotic fractures.

Dual-energy X-ray absorptiometry (DXA) is commonly used to measure BMD all over the world. According to the criteria reported by the World Health Organization, patients with BMD $T$-scores (mean BMD in young healthy adults from 20 to $40 \mathrm{yr}$ ) of $\leq-2.5$ standard deviation can be diagnosed with osteoporosis. The reference standard from which the $T$-score is calculated is the female, white, age 20-29 yr, NHANES III database.

The Chinese population is ethnically diverse. In general, people from the northern part of China are taller and heavier than those from the southern part. In a previous nationwide, multicenter DXA study, Cheng et al $(1,2)$ found that the mean BMD values of the spine and hip were higher in women from the northern part of China than in women from the southern part (the male data were not published). However, the BMD values obtained from DXA data were areal bone mineral density (aBMD) values and were bone size dependent. In addition, the 2-dimensional nature of DXA might not reflect the real differences in BMD values among the people with different body shapes.

Quantitative computed tomography (QCT) allows measurement of volumetric bone mineral density (vBMD) values without superimpositions of the cortical bone and the other tissues. With the development in computed tomography (CT) technology, QCT has been increasingly performed in our clinical practice. Moreover, compared to DXA, QCT also makes it possible to assess both anatomical morphology and vBMD with a single scan, without causing pain due to movement in the patients with fractures (3). Until now, the QCT has been regarded as a surrogate to DXA in BMD measurement, especially for the elderly and for people with fractures.

Although QCT has a relatively higher dose of radiation $(0.06-2.9 \mathrm{mSv})$, it is considered to be helpful in accurately evaluating bone status in young adults. Moreover, during DXA measurement, the superimposed soft tissues of obese patients (the body mass index [BMI] $>25 \mathrm{~kg} /$ $\mathrm{m}^{2}$ ) will make the aBMD values larger because of the attenuation of the X-ray beams and the beam-hardening artifact (4). However, there is still no study that has compared these 2 modalities in measuring the BMD values of the young, healthy Chinese population. Therefore, the present study was performed to compare spinal vBMD with spinal aBMD among young adults from 3 eastern provincial capital cities in Mainland China.

\section{Materials and Methods}

\section{Participants}

A total of 416 young healthy adults (age range: 20 $40 \mathrm{yr}$ ) were recruited from 3 provincial capital cities (Beijing, Shanghai, and Guangzhou) in Mainland China (Table 1).

Table 1

Population Number of Age Groups in Geographically Different Centers

\begin{tabular}{|c|c|c|c|c|c|c|}
\hline \multirow[b]{2}{*}{ Center } & \multirow[b]{2}{*}{ Age (yr) } & \multicolumn{2}{|c|}{ QCT } & \multicolumn{2}{|c|}{ DXA } & \multirow[b]{2}{*}{ Total } \\
\hline & & Men & Women & Men & Women & \\
\hline \multirow[t]{5}{*}{ Beijing } & $20-25$ & 13 & 6 & 15 & 7 & 41 \\
\hline & $26-30$ & 17 & 34 & 19 & 28 & 98 \\
\hline & $31-35$ & 27 & 30 & 18 & 29 & 104 \\
\hline & $36-40$ & 29 & 26 & 34 & 31 & 120 \\
\hline & Sum & 86 & 96 & 86 & 95 & 363 \\
\hline \multirow[t]{5}{*}{ Shanghai } & $20-25$ & 11 & 8 & 16 & 11 & 46 \\
\hline & $26-30$ & 10 & 15 & 9 & 11 & 45 \\
\hline & $31-35$ & 9 & 23 & 14 & 19 & 65 \\
\hline & $36-40$ & 17 & 13 & 13 & 18 & 61 \\
\hline & Sum & 47 & 59 & 52 & 59 & 217 \\
\hline \multirow{5}{*}{ Guangzhou } & $20-25$ & 11 & 13 & 6 & 13 & 43 \\
\hline & $26-30$ & 15 & 20 & 20 & 26 & 81 \\
\hline & $31-35$ & 11 & 15 & 16 & 10 & 52 \\
\hline & $36-40$ & 23 & 20 & 18 & 19 & 80 \\
\hline & Sum & 60 & 68 & 60 & 68 & 256 \\
\hline Sum & & 193 & 223 & 198 & 222 & 836 \\
\hline Total & & \multicolumn{2}{|c|}{416} & \multicolumn{2}{|c|}{420} & \\
\hline
\end{tabular}

Abbr: DXA, dual-energy X-ray absorptiometry; QCT, quantitative computed tomography. 
All participants underwent CT scans of the lumbar spine for medical purposes, such as trauma or acute abdominal pain. Then, the participants were screened with a health questionnaire. Subjects with chronic diseases known to affect bone metabolism, such as diseases of the heart, kidney, liver, and thyroid, parathyroid, or adrenal glands; those with diabetes mellitus; and those with a history of a malignant tumor were excluded. In addition, those who were taking medications (e.g., glucocorticoids, anticonvulsants, fluoride, bisphosphonates, thyroxin, and estrogen replacement therapy) known to affect bone metabolism were also excluded. Informed consent was obtained from all participants before QCT measurement. The present study was approved by the ethics review board of Beijing Jishuitan Hospital.

\section{QCT Measurements}

QCT images were acquired with an Aquilion 64-slice CT scanner (Toshiba Medical Systems, Tokyo, Japan) in Beijing (from Beijing Jishuitan Hospital), with a GE 64-slice LightSpeed volume CT scanner (GE Healthcare, Madison, WI) in Shanghai (from Shanghai 10th People's Hospital), and with a Siemens Somatom Definition 64-slice CT scanner (Siemens AG, Erlangen, Germany) in Guangzhou (from the General Hospital of Guangzhou Military Command of People's Liberation Army). Mindways QCT Pro system (Mindways Software Inc., Austin, TX) was used in all 3 medical centers. Mindways quality assurance and CT calibration phantoms $\left(50,100,200 \mathrm{mg} / \mathrm{cm}^{3}\right)$ were employed in all 3 cities. These were solid phantoms calibrated to the standard aqueous $\mathrm{K}_{2} \mathrm{HPO}_{4} \mathrm{BMD}$. Each CT scanner was first characterized with the scan of the Mindways torso quality assurance phantom on top of the calibration phantom. The tube voltage was the same $(120 \mathrm{kVp})$ in all the 3 cities, whereas the tube current differed slightly. A single European Spine Phantom (ESP-145) was sent to each center and scanned 10 times with repositioning for cross-calibration. The ESP phantoms were designed to simulate the "average" resident in Europe rather than the average resident of the studied metropolitan cities in China.

After phantom experiments, the Mindways calibration phantom was placed under the back of the subject and scanned simultaneously. Vertebrae from L1 to L4 were scanned with the subject in a supine position. All QCT images were transferred to Beijing (Beijing Jishuitan Hospital) and underwent further analyses. QCT Pro analysis software was used to place elliptical regions of interest in the middle plane of 3 vertebral bodies (L2-L4) in the trabecular bone, avoiding the cortical bone of the vertebrae.

\section{BMD Data Obtained via DXA}

In our previous nationwide multicenter large-scale DXA study, 1655 healthy young adults $(20-40 \mathrm{yr})$ were recruited from Beijing, Guangzhou, Shanghai, Nanjing, Jiaxing, and Chengdu. For all the participants, the lumbar spine was scanned with GE Lunar (GE Healthcare, Waukesha, WI) DXA scanners (1). A total of 420 subjects were selected from the DXA database as controls for comparisons, which were matched by gender, age ( $\pm 2 \mathrm{yr})$, height $( \pm 2 \mathrm{~cm})$, weight $( \pm 2 \mathrm{~kg})$, and geographic territory. The DXA measurements were obtained using GE Lunar scanners (GE Healthcare) and were analyzed using the manufacturer's software. The aBMD data referred to the mean value in 4 whole vertebrae (L1-L4, different with vBMD data acquired from L2 to L4 vertebra bodies). The same ESP was scanned 10 times with repositioning in each DXA scanner for cross-calibration.

\section{Statistical Analysis}

SPSS for Windows statistical software (version 18.0; SPSS, Inc., Chicago, IL) was used for statistical analyses. All data were expressed as mean \pm standard deviation. Independent samples $t$-test was used to compare the difference in BMD results measured by QCT with those measured by DXA. One-way analysis of variance was used to compare the differences in BMD values among the 3 geographically diverse centers. Moreover, least significant difference post hoc test was used for pairwise analyses. The Pearson correlation analysis was used to investigate the correlations between the BMI and BMD values. The BMI was calculated as the ratio of weight to height squared (in kilogram per square meter). $p$ values less than 0.05 were considered statistically significant.

\section{Results}

\section{Demographic Data}

The demographic characteristics of the participants from the different centers are presented in Tables 1 and 2. The mean age of the 416 participants in the present study (vBMD group) was $(30.8 \pm 5.5)$ yr (range: $20-40 \mathrm{yr}$ ). The mean height, weight, and BMI of the vBMD group were $(166.3 \pm 7.1) \mathrm{cm},(62.1 \pm 11.4) \mathrm{kg}$, and $(22.4 \pm 3.2) \mathrm{kg} / \mathrm{m}^{2}$, respectively. In addition, the demographic data of the 420 participants in the matched control group from a previous multicenter large-scale DXA aBMD study (the aBMD database of men was unpublished) were summarized as follows: (1) age: $(30.9 \pm 5.6) \mathrm{yr}$, (2) height: $(165.8 \pm 7.4) \mathrm{cm}$, (3) weight: $(62.4 \pm 11.3) \mathrm{kg}$, and (4) BMI: $(22.6 \pm 3.3) \mathrm{kg} / \mathrm{m}^{2}$.

\section{Cross-Calibration of QCT and DXA}

The ESP was kept identical during the QCT and DXA measurement cross-calibration. The precision of aBMD and vBMD measurements obtained on the ESP is showed in Tables 3 and 4 . The data obtained from each center were used in the statistical analysis for further correction.

\section{Geographic and Gender Differences in Volumetric vs $a B M D$}

Generally, both the vBMD and the aBMD values differed significantly among the 3 geographically diverse 
Table 2

Demographic Characteristics by Geographic Centers

\begin{tabular}{|c|c|c|c|c|c|c|c|}
\hline \multirow[b]{2}{*}{ Center } & \multirow[b]{2}{*}{ Parameters } & \multicolumn{3}{|c|}{ QCT } & \multicolumn{3}{|c|}{ DXA } \\
\hline & & Men & Women & Average & Men & Women & Average \\
\hline \multirow[t]{4}{*}{ Beijing } & Age (yr) & $31.4 \pm 5.6$ & $30.8 \pm 5.1$ & $31.1 \pm 5.3$ & $31.7 \pm 6.2$ & $31.6 \pm 4.7$ & $31.6 \pm 5.5$ \\
\hline & Height $(\mathrm{cm})$ & $172.7 \pm 4.7$ & $162.1 \pm 4.6$ & $167.2 \pm 7.0$ & $172.4 \pm 4.8$ & $161.5 \pm 5.5$ & $166.7 \pm 7.5$ \\
\hline & Weight (kg) & $71.9 \pm 10.4$ & $57.7 \pm 8.2$ & $64.5 \pm 11.7$ & $72.1 \pm 10.1$ & $57.6 \pm 8.4$ & $64.5 \pm 11.7$ \\
\hline & BMI & $24.1 \pm 3.3$ & $22.0 \pm 3.2$ & $23.0 \pm 3.4$ & $24.2 \pm 3.2$ & $22.1 \pm 3.3$ & $23.1 \pm 3.4$ \\
\hline \multirow[t]{4}{*}{ Shanghai } & Age (yr) & $30.5 \pm 6.0$ & $30.4 \pm 4.8$ & $30.5 \pm 5.3$ & $29.9 \pm 6.4$ & $31.0 \pm 5.5$ & $30.5 \pm 5.9$ \\
\hline & Height $(\mathrm{cm})$ & $173.4 \pm 5.5$ & $162.4 \pm 4.3$ & $167.3 \pm 7.3$ & $172.7 \pm 4.8$ & $161.4 \pm 4.8$ & $166.7 \pm 7.4$ \\
\hline & Weight (kg) & $71.1 \pm 7.2$ & $54.5 \pm 7.5$ & $61.9 \pm 11.1$ & $69.2 \pm 8.4$ & $54.8 \pm 7.6$ & $61.5 \pm 10.8$ \\
\hline & BMI & $23.7 \pm 2.2$ & $20.6 \pm 2.5$ & $22.0 \pm 2.8$ & $23.2 \pm 2.5$ & $21.0 \pm 2.6$ & $22.0 \pm 2.8$ \\
\hline \multirow[t]{4}{*}{ Guangzhou } & Age (yr) & $31.2 \pm 6.1$ & $30.3 \pm 5.8$ & $30.7 \pm 5.9$ & $30.8 \pm 5.0$ & $29.8 \pm 6.1$ & $30.3 \pm 5.9$ \\
\hline & Height $(\mathrm{cm})$ & $169.6 \pm 4.3$ & $159.3 \pm 4.3$ & $164.1 \pm 6.7$ & $169.6 \pm 4.5$ & $158.9 \pm 4.3$ & $163.9 \pm 6.9$ \\
\hline & Weight (kg) & $65.0 \pm 10.2$ & $53.3 \pm 7.6$ & $58.8 \pm 10.6$ & $65.8 \pm 9.0$ & $55.2 \pm 9.5$ & $60.2 \pm 10.6$ \\
\hline & BMI & $22.6 \pm 3.1$ & $21.0 \pm 2.8$ & $21.7 \pm 3.0$ & $22.9 \pm 3.0$ & $21.9 \pm 3.6$ & $22.3 \pm 3.4$ \\
\hline
\end{tabular}

Abbr: BMI, body mass index; DXA, dual-energy X-ray absorptiometry; QCT, quantitative computed tomography.

Independent sample $t$-test: ${ }^{a} T=-15.05, p<0.01{ }^{b} T=-10.03, p<0.01 ;{ }^{c} T=-4.34, p<0.01 ;{ }^{d} T=-11.11, p<0.01 ;{ }^{e} T=-11.51, p<0.01$; ${ }^{f} T=-6.53, p<0.01 ;{ }^{g} T=-13.53, p<0.01 ;{ }^{h} T=-7.26, p<0.01 ;{ }^{i} T=-2.99, p<0.01 ;{ }^{j} T=-14.32, p<0.01 ;{ }^{k} T=-10.55, p<0.01 ;{ }^{l} T=-4.40$, $p<0.01 ;{ }^{m} T=-12.36, p<0.01 ;{ }^{n} T=-9.54, p<0.01 ;^{o} T=-4.57, p<0.01 ;{ }^{p} T=-13.60, p<0.01 ;{ }^{q} T=-6.44, p<0.01 ;{ }^{r} T=-1.72, p=0.087>0.05$.

centers (vBMD: $F=3.266, p=0.039<0.05$; aBMD: $F=8.916$, $p<0.05)$. Specifically, the vBMD values and the aBMD values of participants from Beijing were both significantly different from those from Guangzhou (vBMD: $p=0.016<0.05$ and aBMD: $p<0.05$, respectively). Moreover, statistically significant differences in aBMD values were also found between participants from Beijing and Shanghai $(p=0.011<0.05)$. However, there were no significant differences in the vBMD values between participants from the above 2 centers $(p=0.836>0.05)$. Furthermore, no significant differences were found between participants from Shanghai and Guangzhou in terms of

Table 3

The Short-Term Precision of vBMD Measurements Obtained on the ESP

\begin{tabular}{lccccccr}
\hline & \multicolumn{3}{c}{ Mean \pm SD $\left(\mathrm{mg} / \mathrm{cm}^{3}\right)$} & & \multicolumn{3}{c}{ CV $(\%)$} \\
\cline { 2 - 4 } Cities & 50 & 100 & 200 & & 50 & 100 & 200 \\
\hline Beijing & $51.2 \pm 0.4$ & $99.7 \pm 0.5$ & $186.0 \pm 0.8$ & & 0.8 & 0.5 & 0.4 \\
Guangzhou & $53.8 \pm 0.4$ & $103.4 \pm 0.6$ & $195.0 \pm 0.8$ & & 0.7 & 0.6 & 0.4 \\
Shanghai & $54.4 \pm 0.5$ & $109.1 \pm 0.5$ & $210.2 \pm 0.9$ & & 1.0 & 0.4 & 0.4 \\
\hline
\end{tabular}

Abbr: ESP, European Spine Phantom; SD, standard deviation; vBMD, volumetric bone mineral density.

Table 4

The Relative Precision of aBMD Measurements Obtained on the ESP

\begin{tabular}{lccccccc}
\hline & \multicolumn{3}{c}{ Mean $\pm \mathrm{SD}\left(\mathrm{g} / \mathrm{cm}^{2}\right)$} & & \multicolumn{3}{c}{$\mathrm{CV}(\%)$} \\
\cline { 2 - 4 } \cline { 7 - 9 } Cities & 0.5 & 1.0 & 1.5 & & 0.5 & 1.0 & 1.5 \\
\hline Beijing & $0.6202 \pm 0.0071$ & $1.1145 \pm 0.0091$ & $1.6054 \pm 0.0202$ & & 1.2 & 0.8 & 1.3 \\
Guangzhou & $0.6105 \pm 0.0088$ & $1.0996 \pm 0.0147$ & $1.5874 \pm 0.0216$ & & 1.4 & 1.3 & 1.4 \\
Shanghai & $0.6117 \pm 0.0093$ & $1.103 \pm 0.0092$ & $1.5801 \pm 0.0106$ & & 1.5 & 0.8 & 0.7 \\
\hline
\end{tabular}

Abbr: aBMD, areal bone mineral density; ESP, European Spine Phantom; SD, standard deviation. 
Table 5

Geographic Differences in Data for vBMD and aBMD

\begin{tabular}{llcrrr}
\hline BMD & Gender & Beijing & Shanghai & Guangzhou & Total \\
\hline \multirow{2}{*}{ vBMD values } & Male & $166.7 \pm 32.0^{a}$ & $165.8 \pm 29.4^{a}$ & $179.0 \pm 32.7^{a}$ & $170.3 \pm 32.0$ \\
& Female & $178.5 \pm 27.7^{b}$ & $180.0 \pm 23.6^{b}$ & $183.2 \pm 28.6^{b}$ & $180.3 \pm 26.9$ \\
& Average & $172.9 \pm 30.3^{c}$ & $173.7 \pm 27.2^{c}$ & $181.2 \pm 30.5^{c}$ & $175.7 \pm 29.8$ \\
aBMD values & Male & $1.14 \pm 0.12^{d}$ & $1.10 \pm 0.12^{d}$ & $1.08 \pm 0.14^{d}$ & $1.11 \pm 0.13$ \\
& Female & $1.17 \pm 0.11^{e}$ & $1.13 \pm 0.12^{e}$ & $1.12 \pm 0.11^{e}$ & $1.14 \pm 0.12$ \\
& Average & $1.16 \pm 0.12^{f}$ & $1.12 \pm 0.12^{f}$ & $1.10 \pm 0.13^{f}$ & $1.13 \pm 0.13$ \\
\hline
\end{tabular}

Abbr: aBMD, areal bone mineral density; ANOVA, analysis of variance; BMD, bone mineral density; LSD, least significant difference; vBMD, volumetric bone mineral density.

${ }^{a}$ One-way ANOVA: $F=3.318, p=0.038<0.05$; LSD post hoc test: Beijing vs Shanghai, $p=0.866>0.05$, Beijing vs Guangzhou, $p=0.022<0.05$, Shanghai vs Guangzhou, $p=0.032<0.05$.

${ }^{b}$ One-way ANOVA: $F=0.603, p=0.548>0.05$.

${ }^{c}$ One-way ANOVA: $F=3.266, p=0.039<0.05$; LSD post hoc test: Beijing vs Shanghai, $p=0.836>0.05$, Beijing vs Guangzhou, $p=0.016<0.05$, Shanghai vs Guangzhou, $p=0.053>0.05$.

${ }^{d}$ One-way ANOVA: $F=4.442, p=0.013<0.05$; LSD post hoc test: Beijing vs Shanghai, $p=0.116>0.05$, Beijing vs Guangzhou, $p=0.004<0.05$, Shanghai vs Guangzhou, $p=0.250>0.05$.

${ }^{e}$ One-way ANOVA: $F=4.713, p=0.010<0.05$; LSD post hoc test: Beijing vs Shanghai, $p=0.038<0.05$, Beijing vs Guangzhou, $p=0.004<0.05$, Shanghai vs Guangzhou, $p=0.520>0.05$.

${ }^{f}$ One-way ANOVA: $F=8.916, p<0.001$; LSD post hoc test: Beijing vs Shanghai, $p=0.011<0.05$, Beijing vs Guangzhou, $p<0.001$, Shanghai vs Guangzhou, $p=0.204>0.05$.

the aBMD values $(p=0.204>0.05)$ and the vBMD values $(p=0.053>0.05$; Table 5$)$.

Further analyses were performed based on gender. Specifically, statistical significant differences in both the vBMD values $(p=0.022<0.05)$ and the aBMD values $(p=0.004<0.05)$ were found between the male participants from Beijing and Guangzhou. However, no significant differences in both the vBMD values $(p=0.866>0.05)$ and the aBMD values $(p=0.116>0.05)$ were found between the male participants from Beijing and Shanghai. Moreover, a statistically significant difference in $\mathrm{vBMD}$ values was also found between the male participants from Shanghai and Guangzhou $(p=0.032<0.05)$. However, there were no differences in the aBMD values between the male participants from the above 2 centers. Interestingly, the situations were quite different for the female participants. Although the vBMD values of the 3 centers showed no significant differences $(F=0.603, p=0.548>0.05)$, the aBMD values among the 3 centers were significantly different $(F=4.713, p=0.010<0.05)$.

A significant difference between genders was found in the overall analysis of the vBMD values $(T=-3.474$, $p=0.001<0.05$ ), and the vBMD values of the women were $5.9 \%$ higher than those of the men (CV of vBMD values: men, $18.8 \%$; women, $14.9 \%)$. The vBMD values of the women were $7.1 \%, 8.6 \%$, and $2.3 \%$ higher than those of the men, from Beijing $(p<0.01)$, Shanghai $(p<0.01)$, and Guangzhou ( $p=0.442>0.05$; the CV of vBMD values in the participants from Beijing, Shanghai, and Guangzhou were $19.2 \%, 17.7 \%$, and $18.3 \%$, respectively, for men, and $15.5 \%, 13.1 \%$, and $15.6 \%$, respectively, for women). However, no significant differences in the aBMD values between genders were found among the 3 centers (Beijing: $T=1.797$, $p=0.074>0.05$; Shanghai: $T=1.159, p=0.249>0.05$; Guangzhou: $T=1.845, p=0.067>0.05)$. The BMD values of the different age groups are presented in Table 6 .

Table 6

BMD Values for Different Age Groups

\begin{tabular}{|c|c|c|c|c|}
\hline \multirow[b]{2}{*}{ Age (yr) } & \multicolumn{2}{|c|}{ aBMD $\left(\mathrm{g} / \mathrm{cm}^{2}\right)$} & \multicolumn{2}{|c|}{$\mathrm{vBMD}\left(\mathrm{mg} / \mathrm{mL}^{3}\right)$} \\
\hline & Men & Women & Men & Women \\
\hline $20-25$ & $1.1339 \pm 0.1267$ & $1.1063 \pm 0.0970$ & $181.4 \pm 27.7$ & $179.5 \pm 27.8$ \\
\hline $26-30$ & $1.1545 \pm 0.1183$ & $1.1540 \pm 0.1114$ & $170.7 \pm 32.1$ & $182.1 \pm 22.5$ \\
\hline $31-35$ & $1.0726 \pm 0.1293$ & $1.1600 \pm 0.1236$ & $172.4 \pm 32.0$ & $182.6 \pm 29.8$ \\
\hline $36-40$ & $1.0883 \pm 0.1354$ & $1.1443 \pm 0.1302$ & $159.5 \pm 32.4$ & $175.9 \pm 27.9$ \\
\hline
\end{tabular}

Abbr: aBMD, areal bone mineral density; BMD, bone mineral density; vBMD, volumetric bone mineral density. 


\section{Relationship between BMI and BMD}

A statistically significant positive linear correlation was found between the aBMD values and the BMI in women $(r=0.297, p<0.01)$ but not in men $(r=0.137$, $p=0.055>0.05)$. In addition, such correlation was not found between the vBMD values and the BMI in both genders $(r=-0.081, p=0.265>0.05$ for men; $r=0.034$, $p=0.614>0.05$ for women $)$.

\section{Discussion}

BMD measurements play an important role in the clinical diagnosis of osteoporosis. There are 2 types of methods: (1) DXA, which is predominantly used for aBMD measurements, and (2) QCT, which is increasingly performed for vBMD measurements. Previous studies have focused on the comparisons between DXA and QCT applications (5-9), but few have been performed in healthy young adults. In the present study, the aBMD and vBMD values of healthy young adults from 3 geographically diverse cities of Mainland China were compared. It is believed that the results of the present study provide a normal reference database of the vBMD values for the healthy young Chinese population. Because of the ethnic genetically diversities and environmental dissimilarity among people from geographically different areas, establishing the normal vBMD reference database focusing on the Chinese population is necessary for the clinical diagnosis of osteoporosis.

Our study demonstrated that the vBMD values of young women were higher than those of young men. The genderrelated differences in vBMD values were found in participants from both Beijing and Shanghai. These results seemed to be inconsistent with the findings of previous studies, which showed a race-specific but gender-independent effect in puberty and adulthood on the vBMD values (10-12). However, Kelly et al reported that men had lower lumbar spine BMD values than their female cotwins in a research performed in dizygotic twins (13). Karantanas et al stated that the vBMD values in healthy adult Greek women less than $37 \mathrm{yr}$ of age were higher than those in men (14). Compston et al found that the vBMD values in healthy adult women in the United Kingdom less than 35 yr of age were higher than those in men (15). Early falling in vBMD values among young men that was hormone-independent might be caused by the increase in total bone area with constant bone mineral content.

In our study, the aBMD values in young adults were remarkably different among the participants from the 3 geographically diverse centers, with northerners (participants from Beijing, who were taller and heavier) showing higher aBMD values than southerners (participants from Guangzhou). However, the vBMD values of Chinese northerners were lower than those of Chinese southerners (Table 5). Considering that the DXA determines the aBMD in 2-dimensional regions of interest with integral trabecular and cortical bone measurement, it may be concluded that the differences seen in DXA may be a function of both cortical bone and bone size.

We found significant differences in the aBMD values merely between the young adults from Beijing and Guangzhou. However, the vBMD values of young men from Guangzhou differed significantly from those of young men from the other 2 cities (Table 5). We attributed the inconsistency between the vBMD and the aBMD findings mainly to the technological differences between the DXA and QCT measurements: the former was a 2-dimension-based technique, whereas the latter was an accurate 3-dimensionbased method. This difference meant that regional heterogeneity should be taken into consideration when bone health was evaluated using DXA among different groups of people in China. However, measuring the vBMD values might be appropriate if adjustments for height and BMI are not feasible.

In addition, the artificial nature of the difference between BMD values for people from diverse geographic regions should also be taken into account. The Chinese society is now facing the challenges of an aging population and accelerating urbanization. The globalization of unhealthy lifestyles, such as undernutrition, overnutrition, tobacco use, alcohol use, drug abuse, and sedentary behavior, significantly affects the intake and deposition of calcium and minerals. Other environmental factors, including air pollution and contamination of food and drinking-water supplies, might also affect BMD values. Moreover, hereditary factors also play an important role in leading to primary osteoporosis as well $(16,17)$.

There were 2 main limitations in the present study. First, a comparative study between QCT and DXA performed on the same cohort would have been more efficient and compelling than an age- and gender-matched study. However, the matched-cohort method that we used was also generally accepted for comparative research (18). Second, our sample size was relatively small, especially with regard to the correlative DXA studies. If our sample size had been larger and if a multicenter study had been performed using the same cohort as control, the present study would have provided more convincing information about the use of QCT and DXA in BMD measurements and in the diagnosis of osteoporosis. Third, the rate of vBMD changes usually differs from that of aBMD in any age (Table 6) and the number of our studied subjects in the 20- to 25-yr group was about $60 \%$ of that in the remaining 3 groups. Such factors influence the $\mathrm{CV}$ values and might have some influence on the conclusions reached.

In conclusion, we performed an age- and gender-matched study of a Chinese population to compare the vBMD and aBMD value measurements. The present study demonstrated an overall heterogeneity and a gender-dependent effect in spinal BMD among young adults from 3 eastern provincial capital cities in Mainland China. As a reliable method for BMD measurement and osteoporosis assessment, QCT measurements can improve our knowledge of 
the physiology and the pathophysiology of age-related bone evolution.

\section{Acknowledgments}

This research was supported by the Natural National Science Foundation of China (Support No. 81071131). Medical editor Katharine O'Moore-Klopf, ELS (East Setauket, NY) provided professional English-language editing of this article.

\section{References}

1. Cheng XG, Yang DZ, Zhou Q, et al. 2007 Age-related bone mineral density, bone loss rate, prevalence of osteoporosis, and reference database of women at multiple centers in China. J Clin Densitom 10:276-284.

2. Li N, Li XM, Xu L, et al. 2013 Comparison of QCT and DXA: osteoporosis detection rates in postmenopausal women. Int J Endocrinol 895474.

3. Cheng X, Wang L, Wang Q, et al. 2014 Validation of quantitative computed tomography-derived areal bone mineral density with dual energy X-ray absorptiometry in an elderly Chinese population. Chin Med J 127:14451449.

4. Binkley N, Krueger D, Vallarta-Ast N. 2003 An overlying fat panniculus affects femur bone mass measurement. J Clin Densitom 6:199-204.

5. Mylona M, Leotsinides M, Alexandrides T, et al. 2005 Comparison of DXA, QCT and trabecular structure in beta-thalassaemia. Eur J Haematol 74:430-437.

6. Kleerekoper M, Nelson DA, Flynn MJ, et al. 1994 Comparison of radiographic absorptiometry with dual-energy x-ray absorptiometry and quantitative computed tomography in normal older white and black women. J Bone Miner Res 9:1745-1749.

7. Yu W, Gluer CC, Grampp S, et al. 1995 Spinal bone mineral assessment in postmenopausal women: a comparison between dual X-ray absorptiometry and quantitative computed tomography. Osteoporos Int 5:433-439.

8. Markel MD, Wikenheiser MA, Morin RL, et al. 1990 Quantification of bone healing. Comparison of QCT, SPA, MRI, and DEXA in dog osteotomies. Acta Orthop Scand 61:487498.

9. Engelke K, Libanati C, Liu Y, et al. 2009 Quantitative computed tomography (QCT) of the forearm using general purpose spiral whole-body CT scanners: accuracy, precision and comparison with dual-energy X-ray absorptiometry (DXA). Bone 45:110-118.

10. Kalender WA, Felsenberg D, Louis O, et al. 1989 Reference values for trabecular and cortical vertebral bone density in single and dual-energy quantitative computed tomography. Eur J Radiol 9:75-80.

11. Gilsanz V, Gibbens DT, Roe TF, et al. 1998 Vertebral bone density in children: effect of puberty. Radiology 166:847850.

12. Lu PW, Cowell CT, LLoyd-Jones SA, et al. 1996 Volumetric bone mineral density in normal subjects, aged 5-27 years. J Clin Endocrinol Metab 81:1586-1590.

13. Kelly PJ, Twomey L, Sambrook PN, Eisman JA. 1990 Sex differences in peak adult bone mineral density. J Bone Miner Res 5:1169-1175.

14. Karantanas AH, Kalef-Ezra JA, Glaros DC. 1991 Quantitative computed tomography for bone mineral measurement: technical aspects, dosimetry, normal data and clinical applications. Br J Radiol 64:298-304.

15. Compston JE, Evans WD, Crawley EO, Evans C. 1988 Bone mineral content in normal UK subjects. Br J Radiol 61:631636.

16. Li H, Xie H, Liu W, et al. 2009 A novel microRNA targeting HDAC5 regulates osteoblast differentiation in mice and contributes to primary osteoporosis in humans. J Clin Invest 119:3666-3677.

17. Li CJ, Cheng P, Liang MK, et al. 2015 MicroRNA-188 regulates age-related switch between osteoblast and adipocyte differentiation. J Clin Invest 125:1509-1522.

18. Kaste SC, Tong X, Hendrick JM, et al. 2006 QCT versus DXA in 320 survivors of childhood cancer: association of BMD with fracture history. Pediatr Blood Cancer 47:936-943. 